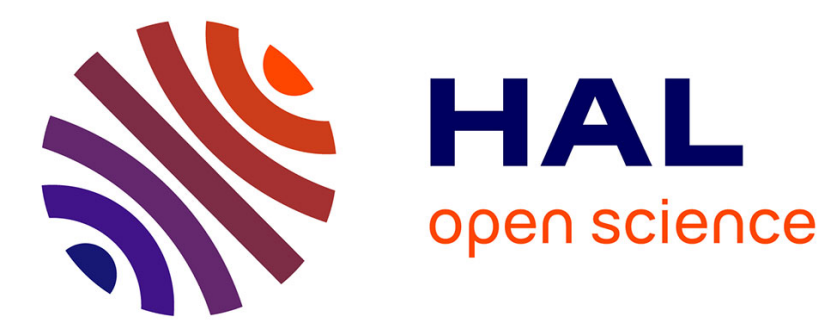

\title{
Subsampling for nonstationary time series with non-zero mean function
}

\author{
Anna Dudek, Łukasz Lenart
}

\section{To cite this version:}

Anna Dudek, Łukasz Lenart. Subsampling for nonstationary time series with non-zero mean function. Statistics and Probability Letters, 2017, 129, pp.252-259. 10.1016/j.spl.2017.06.002 . hal-01415632v4

\section{HAL Id: hal-01415632 \\ https://hal.science/hal-01415632v4}

Submitted on 6 Jul 2017

HAL is a multi-disciplinary open access archive for the deposit and dissemination of scientific research documents, whether they are published or not. The documents may come from teaching and research institutions in France or abroad, or from public or private research centers.
L'archive ouverte pluridisciplinaire HAL, est destinée au dépôt et à la diffusion de documents scientifiques de niveau recherche, publiés ou non, émanant des établissements d'enseignement et de recherche français ou étrangers, des laboratoires publics ou privés. 


\title{
Subsampling for nonstationary time series with non-zero mean function
}

\author{
Anna E. Dudek ${ }^{1,2}$ and Eukasz Lenart ${ }^{3}$ \\ ${ }^{1}$ Institut de Recherche Mathématique de Rennes, \\ Université Rennes 2, Rennes, France, \\ 2 AGH University of Science and Technology, \\ Department of Applied Mathematics, \\ al. Mickiewicza 30, 30-059 Krakow, Poland, \\ email: aedudek@agh.edu.pl \\ ${ }^{3}$ Department of Mathematics, Cracow University of Economics, \\ ul. Rakowicka 27, 31-510 Cracow, Poland, \\ email: lenartl@uek.krakow.pl
}

\begin{abstract}
In this paper a subsampling approach for nonstationary time series with a non-zero mean function is proposed. It is applied for periodically and almost periodically processes. Two statistical tests are constructed. An example with real data is presented.

Keywords: almost periodically correlated, autocovariance function, Fourier coefficients, periodicity, testing

MSC 2010: 62G09, 62G10, 62M10
\end{abstract}

\section{Introduction and problem formulation}

In this paper we discuss a subsampling approach for nonstationary time series, which have a non-zero mean function.

First we introduce some notation. Let $\left\{X_{t}, t \in \mathbb{Z}\right\}$ be a nonstationary time series and $\mu(t)=\mathrm{E}\left(X_{t}\right)$ be its mean function. We assume that $\mu(t) \not \equiv 0$. Moreover, we consider the following decomposition of the mean function

$$
\mu(t)=\mu_{1}(t)+\mu_{2}(t) .
$$

Functions $\mu_{1}(t)$ and $\mu_{2}(t)$ can be interpreted differently depending on the application. In economics they can represent e.g., seasonal or business fluc- 
tuations or a long-term trend, while in telecommunications these can be two periodic functions e.g., the first one may be a pilot tone and the latter one the mean function of some periodic signal. A pilot tone is a signal transmitted for control, synchronization or reference purposes. In the following we assume that $\mu_{1}(t)$ is non-zero and we know how to estimate it. On the other hand, $\mu_{2}(t)$ may be a zero function and moreover we may not have complete knowledge of its form and properties. Such a model describes a real data situation in which only partial information about the mean function is available e.g., we know the nature of some structure contained in the data like seasonality, but other components require further investigation.

We denote the observed sample by $\mathbf{X}_{n}=\left(X_{1}, X_{2}, \ldots, X_{n}\right)$. Let $\hat{\mu}_{1, n}(t)$ be the estimator of $\mu_{1}(t)$ based on $\mathbf{X}_{n}$. We consider the modified sample $\tilde{\mathbf{X}}_{n}=\left(\tilde{X}_{1, n}, \tilde{X}_{2, n}, \ldots, \tilde{X}_{n, n}\right)$, where

$$
\tilde{X}_{t, n}=X_{t}-\hat{\mu}_{1, n}(t) .
$$

In the sequel, for the sake of simplicity we skip the subscript $n$ and we denote elements of the triangular array $\tilde{\mathbf{X}}_{n}$ by $\tilde{X}_{i}, i=1, \ldots, n$. From now we will be using only the sample $\tilde{\mathbf{X}}_{n}$ i.e., we consider partially or entirely demeaned data. This corresponds to the standard procedures of data analysis in which the data is assumed to be demeaned before any further analysis (e.g. secondorder) is performed.

In the next section we present how the subsampling idea for nonstationary time series (see chapter 4 in Politis et al., 1999) can be adapted to our setting. In Section 3 we construct two tests designed for time series with periodic and almost periodic structure. The first one is devoted to the firstorder frequency detection, while the second one can be used for testing the second-order periodicity. Finally, Section 4 contains a real data application of our results.

\section{Subsampling}

In the sequel we use notation introduced by Politis et al., 1999.

Let $\theta=\theta(\mathcal{P}) \in \mathbb{R}$ be the parameter of interest, where $\mathcal{P}$ is the joint probability law governing $\left\{X_{t}, t \in \mathbb{Z}\right\}$. Parameter $\theta$ can simply relate to $\mu_{2}(t)$, but one may consider other characteristics of $X_{t}$. Moreover, let $\hat{\theta}_{n}=\hat{\theta}_{n}\left(\tilde{\mathbf{X}}_{n}\right)$ be the estimator of $\theta$ and $\hat{\theta}_{n, b, t}=\hat{\theta}_{n, b, t}\left(\tilde{X}_{t}, \tilde{X}_{t+1}, \ldots, \tilde{X}_{t+b-1}\right)$ be the estimator of $\theta$ based on subsample $\tilde{X}_{t}, \tilde{X}_{t+1}, \ldots, \tilde{X}_{t+b-1}$. In addition, let $\hat{\theta}_{n, b, t}^{\prime}=\hat{\theta}_{n, b, t}\left(X_{t}^{\prime}, X_{t+1}^{\prime}, \ldots, X_{t+b-1}^{\prime}\right)$, where $X_{i}^{\prime}=X_{i}-\mu_{1}(i), i \in \mathbb{Z}$.

By $J_{b, t}^{\prime}(P)$ and $J_{n}(P)$ we denote the sampling distributions of $\tau_{b}\left(\hat{\theta}_{n, b, t}^{\prime}-\theta\right)$ 
and $\tau_{n}\left(\hat{\theta}_{n}-\theta\right)$, where $\tau_{(\cdot)}$ is an appropriate normalizing sequence. The corresponding cumulative distribution functions are defined as

$$
J_{b, t}^{\prime}(x, P)=P\left\{\tau_{b}\left(\hat{\theta}_{n, b, t}^{\prime}-\theta\right) \leq x\right\}
$$

and

$$
J_{n}(x, P)=P\left\{\tau_{n}\left(\hat{\theta}_{n}-\theta\right) \leq x\right\} .
$$

Finally, following Politis et al., 1999 we assume that $X_{t}$ is $\alpha$-mixing.

Conditions below are sufficient conditions for the subsampling consistency.

Assumption 2.1 There exists a limiting law $J(P)$ such that

(i) $J_{n}(P)$ converges weakly to $J(P)$ as $n \longrightarrow \infty$.

(ii) For any continuity point $x$ of $J(P)$ and for any sequences $n, b$ with $n, b \longrightarrow \infty$ and $b / n \longrightarrow 0$, we have $\frac{1}{n-b+1} \sum_{t=1}^{n-b+1} J_{b, t}^{\prime}(x, P) \longrightarrow$ $J(x, P)$.

$O R$

For any continuity point $x$ of $J(\cdot, P)$ and for any index sequence $\left\{t_{b}\right\}$ we have that $J_{b, t_{b}}^{\prime}(x, P) \longrightarrow J(x, P)$ as $b \longrightarrow \infty$.

(iii) $\max _{1 \leq t \leq n-b+1} \tau_{b}\left|\hat{\theta}_{n, b, t}-\hat{\theta}_{n, b, t}^{\prime}\right| \stackrel{p}{\longrightarrow} 0$.

One may notice that comparing to Politis et al., 1999 (see Assumption 4.2.1. in Politis et al., 1999) we need an additional assumption (iii). It is caused by the fact that in contrast to Politis et al., 1999 we are using partially demeaned data. Finally, condition $(i)$ is identical to the one proposed by Politis et al., while (ii) is adjusted to our problem.

The approximation of $J_{n}(x, P)$ is defined as

$$
\tilde{L}_{n, b}(x)=\frac{1}{n-b+1} \sum_{t=1}^{n-b+1} \mathbf{1}\left\{\tau_{b}\left(\hat{\theta}_{n, b, t}-\hat{\theta}_{n}\right) \leq x\right\}
$$

where $\mathbf{1}\{A\}$ is an indicator function of the event $A$.

Below we state the asymptotic validity of subsampling for general statistics.

Theorem 2.1 Assume that $\left\{X_{t}, t \in \mathbb{Z}\right\}$ is $\alpha$-mixing time series. Under Assumption 2.1 and taking $\tau_{b} / \tau_{n} \longrightarrow 0, b=b(n) \longrightarrow \infty$ such that $b / n \longrightarrow 0$ as $n \longrightarrow \infty$, we have 
(i) if $x$ is a continuity point of $J(\cdot, P)$, then $\tilde{L}_{n, b}(x) \stackrel{p}{\longrightarrow} J(x, P)$,

(ii) if $J(\cdot, P)$ is continuous, then $\sup _{x \in \mathbb{R}}\left|\tilde{L}_{n, b}(x)-J(x, P)\right| \stackrel{p}{\longrightarrow} 0$,

(iii) for $\alpha \in(0,1)$, let $c(1-\alpha)=\inf \{x: J(x, P) \geq 1-\alpha\}$ and $\tilde{c}_{n, b}(1-\alpha)=$ $\inf \left\{x: \tilde{L}_{n, b}(x) \geq 1-\alpha\right\}$. If $J(\cdot, P)$ is continuous at point $c(1-\alpha)$, then subsampling confidence intervals are asymptotically consistent i.e.,

$$
P\left(\tau_{n}\left(\hat{\theta}_{n}-\theta\right) \leq \tilde{c}_{n, b}(1-\alpha)\right) \longrightarrow 1-\alpha .
$$

Remark: In our methodology we applied subsampling to partially demeaned data i.e., to $\tilde{\mathbf{X}}_{n}$. However, an alternative approach is possible. One may consider the usual subsampling and use subsamples $\left(X_{t}-\hat{\mu}_{1, n, b, t}(t), X_{t+1}-\right.$ $\left.\hat{\mu}_{1, n, b, t}(t+1), \ldots, X_{t+b-1}-\hat{\mu}_{1, n, b, t}(t+b-1)\right)$, where $\hat{\mu}_{1, n, b, t}(\cdot)$ is the estimator of the function $\mu_{1}(\cdot)$ based on $\left(X_{t}, X_{t+1}, \ldots, X_{t+b-1}\right)$. In such cases the subsampling consistency can be obtained under the sufficient condition presented in Politis et al., 1999 (see Assumption 4.2.1). However, one should be aware that the estimator $\hat{\mu}_{1, n, b, t}(\cdot)$ may have higher variance or bias than $\hat{\mu}_{1, n}(\cdot)$, especially when $b$ is small.

\section{Applications}

In this section we present possible applications of our subsampling approach. In the first subsection we introduce the class of time series with periodic and almost periodic structure. Additionally, we discuss the assumptions that we use in the consecutive subsections in which we provide two tests.

\subsection{PC/APC time series}

In our consideration we focus on periodically correlated (PC) and almost periodically correlated (APC) time series, which are used for modeling signals in many different fields like economics, telecommunication, mechanics, vibroacoustics and hydrology (see e.g. Hurd and Miamee, 2007, Napolitano, 2012, Napolitano, 2016). Time series $X_{t}$ is called $P C$ with period $T$ if it has periodic mean and autocovariance functions, i.e.

$\mu(t)=\mu(t+T) \quad$ and $\quad B(t, \tau)=\operatorname{Cov}\left(X_{t}, X_{t+\tau}\right)=\operatorname{Cov}\left(X_{t+T}, X_{t+\tau+T}\right)$

for each $t, \tau \in \mathbb{Z}$. For more details we refer the reader to [Hurd and Miamee, 2007]. Sometimes it may happen that period length is not known or a considered signal is a composition of two components with incommensurable periods. 
To model such data APC time series are used (see e.g. Napolitano, 2012). They are a generalization of $\mathrm{PC}$ sequences. A time series that has finite second order moments is called $A P C$ if its mean and autocovariance functions are almost periodic in time. Finally, a function $f(t): \mathbb{Z} \longrightarrow \mathbb{R}$ of an integer variable is called almost periodic if for any $\epsilon>0$ there exists an integer $L_{\epsilon}>0$ such that among any $L_{\epsilon}$ consecutive integers there is an integer $p_{\epsilon}$ with the property $\sup _{t \in \mathbb{Z}}\left|f\left(t+p_{\epsilon}\right)-f(t)\right|<\epsilon$ (see Corduneanu, 1989 for more details).

Analysis of PC and APC processes is often performed in the frequency domain. In such cases the following Fourier expansions of the mean and the autocovariance functions are used

$$
\mu(t)=\sum_{\psi \in \Psi} m(\psi) e^{i \psi t}, \quad B(t, \tau)=\sum_{\lambda \in \Lambda_{\tau}} a(\lambda, \tau) e^{i \lambda t}
$$

for any shift $\tau \in \mathbb{Z}$, where

$$
m(\psi)=\lim _{n \rightarrow \infty} \frac{1}{n} \sum_{j=1}^{n} \mu(j) e^{-i \psi j}, \quad a(\lambda, \tau)=\lim _{n \rightarrow \infty} \frac{1}{n} \sum_{j=1}^{n} B(j, \tau) e^{-i \lambda j}
$$

and $\Psi=\{\psi \in[0,2 \pi): m(\psi) \neq 0\}$ and $\Lambda_{\tau}=\{\lambda \in[0,2 \pi): a(\lambda, \tau) \neq 0\}$ are the sets of the frequencies. The expansions (4) hold, if the sets $\Psi$ and $\Lambda_{\tau}, \tau \in \mathbb{Z}$ are finite. In the case of PC time series this condition always holds and additionally, we know that $\Psi, \Lambda_{\tau} \subseteq\{2 k \pi / T, k=0,1, \ldots, T-1\}$ for any $\tau \in \mathbb{Z}$.

Below we present the conditions that are used in the sequel.

Let $\left\{X_{t}, t \in \mathbb{Z}\right\}$ be an $\alpha$-mixing time series. Depending on the considered case we will assume that $X_{t}$ is PC or APC.

A1 There exists a constant $\delta>0$ such that

$$
\sup _{t \in \mathbb{Z}} E\left|X_{t}\right|^{2+\delta}<\infty \quad \text { and } \quad \sum_{k=0}^{\infty} \alpha(k)^{\delta /(2+\delta)}<\infty
$$

A2 $\left\{X_{t}, t \in \mathbb{Z}\right\}$ is uniformly bounded i.e., there exists a constant $M>0$ such that $P\left(\left|X_{t}\right| \leq M\right)=1$ for all $t \in \mathbb{Z}$. Moreover, there exists a constant $\zeta>0$ such that

$$
\sum_{k=0}^{\infty}(k+1)^{2} \alpha(k)^{\zeta /(8+\zeta)}<\infty
$$


A3 For any $\tau_{1}, \tau_{2}, \tau_{3} \in \mathbb{Z}$ the function $\left(t, \tau_{1}, \tau_{2}, \tau_{3}\right) \longrightarrow \mathrm{E}\left(X_{t} X_{t+\tau_{1}} X_{t+\tau_{2}} X_{t+\tau_{3}}\right)$ is periodic in $t$ i.e.,

$$
\mathrm{E}\left(X_{t} X_{t+\tau_{1}} X_{t+\tau_{2}} X_{t+\tau_{3}}\right)=\mathrm{E}\left(X_{t+T} X_{t+\tau_{1}+T} X_{t+\tau_{2}+T} X_{t+\tau_{3}+T}\right) .
$$

\subsection{Testing the significance of a frequency in the mean func- tion}

In this section we assume that $\left\{X_{t}, t \in \mathbb{Z}\right\}$ is an APC time series. Let us recall that we consider $\mu(t)=\mu_{1}(t)+\mu_{2}(t)$ and we know the estimator of $\mu_{1}(t)$. Moreover, functions $\mu_{1}(t)$ and $\mu_{2}(t)$ have the following Fourier expansions $\mu_{1}(t)=\sum_{\psi \in \Psi_{1}} m(\psi) e^{i \psi t}$ and $\mu_{2}(t)=\sum_{\psi \in \Psi_{2}} m(\psi) e^{i \psi t}$, where $\Psi=$ $\Psi_{1} \cup \Psi_{2}$, the set $\Psi_{1}$ is known and $\Psi_{1} \cap \Psi_{2}=\emptyset$. Then the natural estimator of $\mu_{1}(t)$ has the form (see e.g. Hurd and Miamee, 2007)

$$
\hat{\mu}_{1, n}(t)=\sum_{\psi \in \Psi_{1}}\left[\frac{1}{n} \sum_{j=1}^{n} X_{j} e^{-i \psi j}\right] e^{i \psi t} .
$$

Our aim is to test if for some frequency, for which we know that $\psi_{0} \notin \Psi_{1}$, we have that $\psi_{0} \in \Psi_{2}$. This problem can be equivalently expressed in terms of the corresponding Fourier coefficients i.e., we consider $H_{0}:\left|m\left(\psi_{0}\right)\right|=0$ vs $H_{1}:\left|m\left(\psi_{0}\right)\right|>0$. In this case $\theta=\left|m\left(\psi_{0}\right)\right|$ and the test statistics $T_{n}$ based on $\tilde{\mathbf{X}}_{n}$ is of the form

$$
T_{n}=\sqrt{n}\left|\hat{m}_{n}\left(\psi_{0}\right)\right|=\frac{1}{\sqrt{n}}\left|\sum_{j=1}^{n}\left(X_{j}-\hat{\mu}_{1, n}(j)\right) e^{-i \psi_{0} j}\right| .
$$

Large values of $T_{n}$ suggest that $H_{1}$ is true. The quantiles of $T_{n}$ can be approximated using the subsampling method. For that purpose below we state the subsampling validity for $\left|m\left(\psi_{0}\right)\right|$.

Theorem 3.1 Assume that condition A1 is fulfilled. Moreover, let the set $\Psi$ be finite. Then Assumption 2.1 holds for $\theta=\left|m\left(\psi_{0}\right)\right|$ and $\hat{\theta}_{n}=$ $\left|\hat{m}_{n}\left(\psi_{0}\right)\right|=\frac{1}{n}\left|\sum_{j=1}^{n}\left(X_{j}-\hat{\mu}_{1, n}(j)\right) e^{-i \psi_{0} j}\right|$. Therefore Theorem 2.1 ensures that the subsampling based on $\tilde{\mathbf{X}}_{n}$ is consistent i.e., (i)-(iii) in Theorem 2.1 hold. 


\subsection{Testing if $B(t, \tau)$ is periodic}

In this section we discuss the problem of testing periodicity of the autocovariance function in the class of $\mathrm{PC}$ time series with known period $T$. Note that in such a class of time series we have $\mu_{2}(t) \equiv 0$. Moreover, $\hat{\mu}_{1, n}(t)$ is the estimator of the periodic function $\mu(t)$ and is given by formula (5) with $\Psi=\Psi_{1}=\{2 k \pi / T: k=0,1,2, \ldots, T-1\}$. To simplify the notation we will write $\hat{\mu}_{n}(t)$ instead of $\hat{\mu}_{1, n}(t)$. We take $\tau \in \mathbb{Z}$ and we consider the following test $H_{0}: B(t, \tau)$ is constant in $t \in \mathbb{Z}$ vs $H_{1}: B(t, \tau)$ is periodic in $t$ with period $T$. Note that $H_{0}$ can be now equivalently rewritten as $H_{0}:|a(\lambda, \tau)|=0$ for all $\lambda \in\{1,2, \ldots, T-1\}$. Assume that $\tau \geq 0$. The estimator of $a(\lambda, \tau)$ based on $\tilde{\mathbf{X}}_{n}$ has the form

$$
\hat{a}_{n}(\lambda, \tau)=\frac{1}{n} \sum_{t=1}^{n-\tau}\left(X_{t}-\hat{\mu}_{n}(t)\right)\left(X_{t+\tau}-\hat{\mu}_{n}(t+\tau)\right) e^{-i \lambda t} .
$$

Therefore we consider the following test statistics $U_{n}=\sqrt{n} \sum_{\lambda \in \mathcal{A}}\left|\hat{a}_{n}(\lambda, \tau)\right|$, where $\mathcal{A}=\{2 k \pi / T, k=1, \ldots, T-1\}$. Large values of $U_{n}$ suggest that $H_{1}$ is true. To approximate quantiles of $U_{n}$, we show subsampling consistency.

Theorem 3.2 Assume that conditions A2 and A3 are fulfilled. Then Assumption 2.1 holds for $\theta=\sum_{\lambda \in \mathcal{A}}|a(\lambda, \tau)|$ and $\hat{\theta}_{n}=\sum_{\lambda \in \mathcal{A}}\left|\hat{a}_{n}(\lambda, \tau)\right|$, where $\hat{a}_{n}(\lambda, \tau)$ is given by (6). Therefore Theorem 2.1 ensures that the subsampling based on $\tilde{\mathbf{X}}_{n}$ is consistent i.e., (i)-(iii) in Theorem 2.1 hold.

\section{A real data example}

Below we present the real data application of the testing problem described in Section 3.2. For that purpose we use data published by Eurostat, concerning monthly industrial production (percentage change on previous period) for Mining and quarrying; manufacturing; electricity, gas, steam and air conditioning supply in three countries: France, Germany and Italy. In the first row of Figure 1 are visualized gross data i.e., data that were not modified in any way before publishing (neither seasonally nor calendar adjusted). The length of the analyzed sequence for each country is $n=288$ (January 1992 - December 2015).

The considered data are known to contain two types of periodic structures. The first one represents the typical seasonal effect with period length equal to 12 months. The second one is a consequence of the so-called trading-day 
effect or the calendar effect, which is caused by a different number of working days in each month. Note that, each February in non-leap-years has the same number (equal to 20) of working days. For other months, this number varies from month to month. For the Georgian calendar this additional deterministic source of variability (well characterized for macroeconomic time series) is periodic with period equal to 400 years. But only a few frequencies corresponding to this periodic phenomenon are predominant (for more details see chapter 11.2 in Bell et al., 2012). Thus, following the notation introduced in Section 3.2 we have that $\Psi_{1} \subseteq\{2 k \pi / 12, k=0, \ldots, 11\}$. Moreover, it is known that for monthly data the predominant trading-day frequency $\psi_{0} \cong 2.18733$.

We perform the test introduced in Section 3.2 to confirm that the frequency $\psi_{0}$ is significant i.e., that $\left|m\left(\psi_{0}\right)\right| \neq 0$. Thus, we test the hypothesis: $\psi_{0} \in \Psi_{2}$ against $\psi_{0} \notin \Psi_{2}$. Note that $\psi_{0} \in \Psi_{2}$ is equivalent to $\left|m\left(\psi_{0}\right)\right| \neq 0$. In our approach we are using data obtained after removing the seasonal effect. One may note that in the case of the raw data, the $\psi_{0}$ is very close to the frequency $\psi=2 \pi / 3 \in \Psi_{1}$. To visualize this fact in the second and in the third row of Figure 1 we present the estimated values of $T_{n}=\sqrt{n}\left|\hat{m}_{n}(\psi)\right|, \psi \in[0, \pi]$ obtained using the raw data $\mathbf{X}_{n}$ and partially demeaned data $\tilde{\mathbf{X}}_{n}$, respectively. The observed effect could have a serious impact on the results of our test if we used simply the raw data. To check that we decided to apply our methodology not only to the data obtained after removing the seasonal effect $\tilde{\mathbf{X}}_{n}$, but also to the raw data $\mathbf{X}_{n}$ (based on Lenart, 2013). Below we discuss results of both approaches.

We set the significance level $\alpha=5 \%$ and subsample length $b \in\{15,20, \ldots, 40\}$. The obtained values of the test statistics for the raw data are: 0.397 (Germany), 0.371 (France) and 0.497 (Italy). In the case of partially demeaned data the corresponding values are a bit lower: 0.382 (Germany), 0.303 (France) and 0.411 (Italy). For each considered country values of the subsampling quantiles are the highest for $b=15$ and decrease when $b$ increases. For example in the case of Germany taking $b=15$ we got a subsampling quantile equal to 0.117 (raw data) and 0.06 (partially demeaned data). Corresponding values for $b=40$ are: 0.094 and 0.042 . Moreover, the raw data quantiles have much higher values than those for the partially demeaned data. As a result in the case of Italy values of the subsampling quantiles for the raw data are close to the values of the test statistics. Additionally, for Italy when the raw data and $b=15$ are considered, the null hypothesis is not rejected i.e., at a significance level of $5 \%$, frequency $\psi_{0}$ is not detected (p-value 0.0511$)$. For $b \in\{20,25,30,35,40\}$ the corresponding $\mathrm{p}$-values are equal to $0.026,0.0114,0,0,0$ and hence $H_{0}$ is rejected. Simultaneously for 
the partially demeaned data, independently of the chosen country and the subsample length $b$, the null hypothesis is strongly rejected ( $\mathrm{p}$-values are equal to 0 ), which means that at the considered significance level we always detect the frequency $\psi_{0}$. Thus, using our subsampling approach allows for better frequency detection.
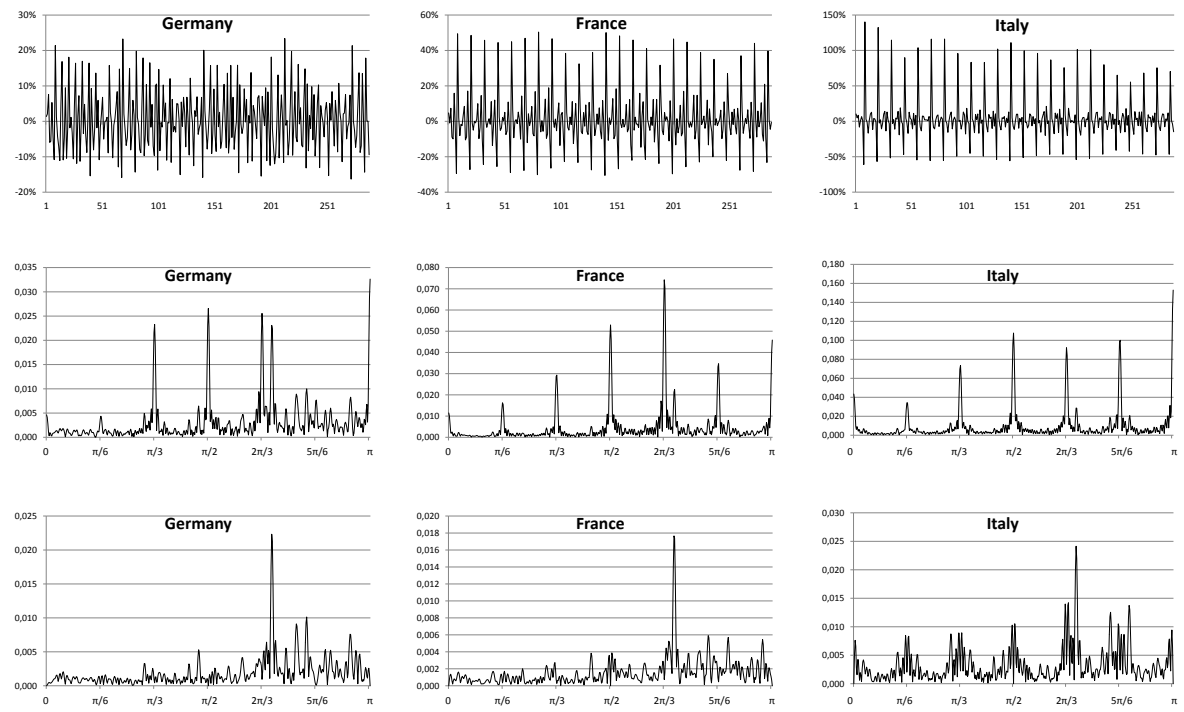

Figure 1: First row: Monthly industrial production (percentage change on previous period, raw data - neither seasonally nor calendar adjusted, source: Eurostat) from Jan. 1992 to Dec. 2015. Second and third rows: values of test statistics $T_{n}$ obtained respectively for the raw data $\mathbf{X}_{n}$ and for data got after subtracting estimates of seasonal means $\tilde{\mathbf{X}}_{n}$.

\section{Appendix}

Proof of Theorem 2.1. The proof is similar to the one proposed by Politis et al., 1999 and hence we skip most of the details, indicating only some major differences. Let $q=n-b+1$ and $U_{n}(x)=1 / q \sum_{t=1}^{q} \mathbf{1}\left\{\tau_{b}\left(\hat{\theta}_{n, b, t}^{\prime}-\right.\right.$ $\theta) \leq x\}$. Notice that $\tilde{L}_{n, b}(x)=1 / q \sum_{t=1}^{q} \mathbf{1}\left\{\tau_{b}\left[\hat{\theta}_{n, b, t}^{\prime}-\theta\right]+\tau_{b}\left[\hat{\theta}_{n, b, t}-\hat{\theta}_{n, b, t}^{\prime}\right]-\right.$ $\left.\tau_{b}\left[\hat{\theta}_{n}-\theta\right] \leq x\right\}$. We show that for any $x \in \mathbb{R}$ and $\epsilon>0$

$$
U_{n}(x-\epsilon) \mathbf{1}\left\{E_{n}\right\} \leq \tilde{L}_{n, b}(x) \mathbf{1}\left\{E_{n}\right\} \leq U_{n}(x+\epsilon),
$$


where $E_{n}=\left\{\max _{1 \leq t \leq q}\left|S_{n, b, t}\right| \leq \epsilon\right\}$ and $S_{n, b, t}=\tau_{b}\left(\left[\hat{\theta}_{n, b, t}-\hat{\theta}_{n, b, t}^{\prime}\right]-\left[\hat{\theta}_{n}-\theta\right]\right)$ for $1 \leq t \leq q$. If $\mathbf{1}\left\{E_{n}\right\}=0$, then (7) is obvious. If $\mathbf{1}\left\{E_{n}\right\}=1$ then

$$
\max _{1 \leq t \leq q}\left|S_{n, b, t}\right|=\tau_{b} \max _{1 \leq t \leq q}\left|\left[\hat{\theta}_{n, b, t}-\hat{\theta}_{n, b, t}^{\prime}\right]-\left[\hat{\theta}_{n}-\theta\right]\right| \leq \epsilon,
$$

which means that uniformly in $1 \leq t \leq q$ we have $S_{n, b, t} \in[-\epsilon, \epsilon]$. Thus, for any $1 \leq t \leq q$ we get that

$$
\begin{gathered}
\mathbf{1}\left\{\tau_{b}\left(\hat{\theta}_{n, b, t}^{\prime}-\theta\right) \leq x-\epsilon\right\} \leq \mathbf{1}\left\{\tau_{b}\left(\hat{\theta}_{n, b, t}^{\prime}-\theta\right) \leq x-S_{n, b, t}\right\} \\
=\mathbf{1}\left\{\tau_{b}\left[\theta_{n, b, t}^{\prime}-\theta\right]+\tau_{b}\left[\hat{\theta}_{n, b, t}-\hat{\theta}_{n, b, t}^{\prime}\right]-\tau_{b}\left[\hat{\theta}_{n}-\theta\right] \leq x\right\}, \\
\mathbf{1}\left\{\tau_{b}\left(\hat{\theta}_{n, b, t}^{\prime}-\theta\right) \leq x+\epsilon\right\} \geq \mathbf{1}\left\{\tau_{b}\left(\hat{\theta}_{n, b, t}^{\prime}-\theta\right) \leq x-S_{n, b, t}\right\} \\
=\mathbf{1}\left\{\tau_{b}\left[\theta_{n, b, t}^{\prime}-\theta\right]+\tau_{b}\left[\hat{\theta}_{n, b, t}-\hat{\theta}_{n, b, t}^{\prime}\right]-\tau_{b}\left[\hat{\theta}_{n}-\theta\right] \leq x\right\} .
\end{gathered}
$$

Summing inequalities (8), (9) over $t=1,2, \ldots, q$ we get (7).

In the next step we show that $P\left(E_{n}\right) \rightarrow 1$. Note that

$$
\max _{1 \leq t \leq q}\left|S_{n, b, t}\right| \leq \max _{1 \leq t \leq q} \tau_{b}\left|\hat{\theta}_{n, b, t}-\hat{\theta}_{n, b, t}^{\prime}\right|+\tau_{b}\left|\hat{\theta}_{n}-\theta\right| .
$$

Using assumptions $(i i i)$ and $(i)$ we obtain that $\max _{1 \leq t \leq q} \tau_{b}\left|\hat{\theta}_{n, b, t}-\hat{\theta}_{n, b, t}^{\prime}\right| \stackrel{p}{\longrightarrow} 0$ and $\tau_{b}\left|\hat{\theta}_{n}-\theta\right| \stackrel{p}{\rightarrow} 0$. Therefore $\max _{1 \leq t \leq q}\left|S_{n, b, t}\right| \stackrel{p}{\longrightarrow} 0$ and we get that $P\left(E_{n}\right) \rightarrow 1$.

Proof of Theorem 3.1. Notice that under A1 the condition (ii) of Assumption 2.1 holds by the same arguments as were used in the proof of Theorem 2.2 in Lenart, 2013. To show $(i)$ note that

$$
\begin{aligned}
& \frac{1}{n} \sum_{t=1}^{n}\left(X_{t}-\hat{\mu}_{1, n}(t)\right) e^{-i \psi_{0} t}-m\left(\psi_{0}\right)=\left(\frac{1}{n} \sum_{t=1}^{n}\left(X_{t}-\mu_{1}(t)\right) e^{-i \psi_{0} t}-m\left(\psi_{0}\right)\right) \\
& +\frac{1}{n} \sum_{t=1}^{n}\left(\mu_{1}(t)-E\left[\hat{\mu}_{1, n}(t)\right]\right) e^{-i \psi_{0} t}+\frac{1}{n} \sum_{t=1}^{n}\left(E\left[\hat{\mu}_{1, n}(t)\right]-\hat{\mu}_{1, n}(t)\right) e^{-i \psi_{0} t} .
\end{aligned}
$$

The second and the third summand on the right-hand side we denote by $p_{n}$ and $q_{n}$, respectively. We have

$$
\begin{aligned}
& \sqrt{n} p_{n}=\frac{1}{\sqrt{n}} \sum_{t=1}^{n} \sum_{\psi \in \Psi_{1}}\left(m(\psi)-\left[\frac{1}{n} \sum_{j=1}^{n} \sum_{\rho \in \Psi} m(\rho) e^{i \rho j} e^{-i \psi j}\right]\right) e^{i \psi t} e^{-i \psi_{0} t} \\
& =\frac{1}{\sqrt{n}} \sum_{\psi \in \Psi_{1}}\left(\frac{1}{n} \sum_{\rho \in \Psi \backslash\{\psi\}} m(\rho) \frac{e^{i(\rho-\psi)}\left(e^{i n(\rho-\psi)}-1\right)}{e^{i(\rho-\psi)}-1}\right) \frac{e^{i\left(\psi-\psi_{0}\right)}\left(e^{i n\left(\psi-\psi_{0}\right)}-1\right)}{e^{i\left(\psi-\psi_{0}\right)}-1} .
\end{aligned}
$$


Therefore $\left|\sqrt{n} p_{n}\right|=O\left(n^{-3 / 2}\right)$. Moreover, note that $\left|\sqrt{n} q_{n}\right|$ is equal to

$$
\begin{aligned}
& \left|\frac{1}{\sqrt{n}}\left(\sum_{\psi \in \Psi_{1}}\left[\frac{1}{n} \sum_{j=1}^{n} \mu(j) e^{-i \psi j}\right]-\sum_{\psi \in \Psi_{1}}\left[\frac{1}{n} \sum_{j=1}^{n} X_{j} e^{-i \psi j}\right]\right) \frac{e^{i\left(\psi-\psi_{0}\right)}\left(e^{i n\left(\psi-\psi_{0}\right)}-1\right)}{e^{i\left(\psi-\psi_{0}\right)}-1}\right| \\
& \quad \leq \frac{1}{\sqrt{n}} \sum_{\psi \in \Psi_{1}}\left[\frac{1}{n} \sum_{j=1}^{n}\left|\mu(j)-X_{j}\right|\right] \frac{2}{\left|e^{i\left(\psi-\psi_{0}\right)}-1\right|} .
\end{aligned}
$$

Since $E\left|\mu(j)-X_{j}\right|$ is bounded by some constant independent of $n$ uniformly at $j \in \mathbb{Z}$, we get that $E\left|\sqrt{n} q_{n}\right|=O\left(n^{-1 / 2}\right)$ and finally that $\sqrt{n} q_{n} \stackrel{p}{\longrightarrow} 0$.

To show (iii) we use the inequality ||$x|-| y|| \leq|x-y|$ (which holds for any $x, y \in \mathbb{C})$. Then for any $1 \leq t \leq n-b+1$ the term $\tau_{b}\left|\hat{\theta}_{n, b, t}-\hat{\theta}_{n, b, t}^{\prime}\right|$ can be bounded from above by

$$
\begin{aligned}
& \frac{1}{\sqrt{b}}\left|\sum_{j=t}^{t+b-1}\left(X_{j}-\hat{\mu}_{1, n}(j)\right) e^{-i \psi_{0} j}-\sum_{j=t}^{t+b-1}\left(X_{j}-\mu_{1}(j)\right) e^{-i \psi_{0} j}\right| \\
& \quad=\frac{1}{\sqrt{b}}\left|\sum_{j=t}^{t+b-1}\left(\sum_{\psi \in \Psi_{1}} m(\psi) e^{i \psi j}-\sum_{\psi \in \Psi_{1}}\left[\frac{1}{n} \sum_{k=1}^{n} X_{k} e^{-i \psi k}\right] e^{i \psi j}\right) e^{-i \psi_{0} j}\right| \\
& \leq \frac{1}{\sqrt{b}} \sum_{\psi \in \Psi_{1}}\left|m(\psi)-\frac{1}{n} \sum_{k=1}^{n} X_{k} e^{-i \psi k}\right| \frac{2}{\left|e^{i\left(\psi-\psi_{0}\right)}-1\right|} .
\end{aligned}
$$

Since $\left|m(\psi)-1 / n \sum_{k=1}^{n} X_{k} e^{-i \psi k}\right|$ does not depend on $t$ and tends to 0 in probability as $n \longrightarrow \infty$ for each $\psi \in \Psi_{1}$, we get that (iii) holds.

Proof of Theorem [3.2. Notice that under A2 and A3 the condition (ii) of Assumption 2.1 holds by the same arguments as were used in the proof of Theorems 1 and 2 in Lenart et al., 2008. To show $(i)$ one needs to decompose $\sqrt{n}\left(\hat{\theta}_{n}-\theta\right)$ similarly to 10 and then to show the convergence to 0 in probability of the corresponding terms. For that purpose let us denote $p_{n}=1 / \sqrt{n} \sum_{t=1}^{n-\tau}\left(X_{t}-\mu(t)\right)\left(\mu(t+\tau)-\hat{\mu}_{n}(t+\tau)\right) e^{-i \lambda t}$, $q_{n}=1 / \sqrt{n} \sum_{t=1}^{n-\tau}\left(\mu(t)-\hat{\mu}_{n}(t)\right)\left(X_{t+\tau}-\mu(t+\tau)\right) e^{-i \lambda t}$ and $r_{n}=1 / \sqrt{n} \sum_{t=1}^{n-\tau}(\mu(t)-$ $\left.\hat{\mu}_{n}(t)\right)\left(\mu(t+\tau)-\hat{\mu}_{n}(t+\tau)\right) e^{-i \lambda t}$.

Note that using the same arguments as in the proof of Theorem 2.1 in 
Lenart, 2013 (under the same assumptions) and results of [Kim, 1994 we get that

$$
\left\|\hat{m}_{n}(\psi)-m(\psi)\right\|_{2}=O\left(n^{-1 / 2}\right) .
$$

Thus,

$E\left|p_{n}\right| \leq \sum_{\psi \in \Psi}\left\|m(\psi)-\hat{m}_{n}(\psi)\right\|_{2}\left\|\frac{1}{\sqrt{n}} \sum_{t=1}^{n-\tau}\left(X_{t}-\mu(t)\right) e^{-i \lambda t+i \psi(t+\tau)}\right\|_{2}=o(1)$.

Similarly $E\left|q_{n}\right| \longrightarrow 0$ as $n \longrightarrow \infty$ and hence $p_{n}, q_{n} \longrightarrow 0$ in probability as $n \longrightarrow \infty$. Finally, $E\left|r_{n}\right|$ is bounded by

$$
\begin{aligned}
& \sum_{\psi_{1} \in \Psi} \sum_{\psi_{2} \in \Psi}\left\|m\left(\psi_{1}\right)-\hat{m}_{n}\left(\psi_{1}\right)\right\|_{2}\left\|m\left(\psi_{2}\right)-\hat{m}_{n}\left(\psi_{2}\right)\right\|_{2}\left|\frac{1}{\sqrt{n}} \sum_{t=1}^{n-\tau} e^{-i \lambda t+i \psi_{1} t+i \psi_{2}(t+\tau)}\right| \\
& =\sum_{\psi_{1} \in \Psi} \sum_{\psi_{2} \in \Psi}\left\|m\left(\psi_{1}\right)-\hat{m}_{n}\left(\psi_{1}\right)\right\|_{2}\left\|m\left(\psi_{2}\right)-\hat{m}_{n}\left(\psi_{2}\right)\right\|_{2} O(\sqrt{n})=o(1) .
\end{aligned}
$$

To get ( $i i i)$ note that using similar arguments as in the proof of Theorem 3.1. expression $\tau_{b}\left|\hat{\theta}_{n, b, t}-\hat{\theta}_{n, b, t}^{\prime}\right|$ can be bounded from above by

$$
\begin{aligned}
& \frac{1}{\sqrt{b}} \sum_{\lambda \in \Lambda_{\tau} \backslash\{0\}}\left|\sum_{j=t}^{t+b-1} X_{j}\left[\hat{\mu}_{n}(j+\tau)-\mu(j+\tau)\right] e^{-i \lambda j}\right|+\frac{1}{\sqrt{b}} \sum_{\lambda \in \Lambda_{\tau} \backslash\{0\}} \mid \sum_{j=t}^{t+b-1} X_{j+\tau}\left[\hat{\mu}_{n}(j)\right. \\
& -\mu(j)] e^{-i \lambda j \mid}+\frac{1}{\sqrt{b}} \sum_{\lambda \in \Lambda_{\tau} \backslash\{0\}}\left|\sum_{j=t}^{t+b-1}\left[\hat{\mu}_{n}(j) \hat{\mu}_{n}(j+\tau)-\mu(j) \mu(j+\tau)\right] e^{-i \lambda j}\right|,
\end{aligned}
$$

for any $1 \leq t \leq n-b+1$. Let us denote summands above by $a_{n}, b_{n}$ and $c_{n}$, respectively and $q=n-b+1$. Note that $\max _{1 \leq t \leq q} \tau_{b}\left|\hat{\theta}_{n, b, t_{b}}-\hat{\theta}_{n, b, t}^{\prime}\right| \leq$ $\max _{1 \leq t \leq q} a_{n}+\max _{1 \leq t \leq q} b_{n}+\max _{1 \leq t \leq q} c_{n}$. Thus

$$
\begin{aligned}
\max _{1 \leq t \leq q} a_{n} & \leq \max _{1 \leq t \leq q} \frac{1}{\sqrt{b}} \sum_{\lambda \in \Lambda_{\tau} \backslash\{0\}} \sum_{\psi \in \Psi}\left|\hat{m}_{n}(\psi)-m(\psi)\right|\left|\sum_{j=t}^{t+b-1} X_{j} e^{-i \lambda j+i \psi(j+\tau)}\right| \\
& \leq \frac{1}{\sqrt{b}} \sum_{\lambda \in \Lambda_{\tau} \backslash\{0\}} \sum_{\psi \in \Psi}\left|\hat{m}_{n}(\psi)-m(\psi)\right| M b \text {, a.s., }
\end{aligned}
$$

where the last inequality follows from assumption A2. Hence by (11)

$$
\left\|\max _{1 \leq t \leq q} a_{n}\right\|_{2} \leq \sum_{\psi \in \Psi}\left\|\hat{m}_{n}(\psi)-m(\psi)\right\|_{2} O\left(b^{1 / 2}\right)=O(\sqrt{b / n}) \rightarrow 0 .
$$


This means that $\max _{1 \leq t \leq q} a_{n} \longrightarrow 0$ in probability as $n \longrightarrow \infty$. The corresponding convergence for $b_{n}$ can be obtained analogically. Finally,

$$
\max _{1 \leq t \leq q} c_{n} \leq O(\sqrt{b}) \sum_{\psi_{1} \in \Psi} \sum_{\psi_{2} \in \Psi}\left|\hat{m}_{n}\left(\psi_{1}\right) \hat{m}_{n}\left(\psi_{2}\right)-m\left(\psi_{1}\right) m\left(\psi_{2}\right)\right| .
$$

To finish the proof of the theorem it is enough to show that the each term of the sum on the right-hand side of $(12)$ converges to 0 in probability as $n \longrightarrow \infty$. For that purpose let us take any $\psi_{1}, \psi_{2} \in \Psi$. If $\left|m\left(\psi_{1}\right) m\left(\psi_{2}\right)\right|=0$ (without loss of generality we assume that $\left|m\left(\psi_{1}\right)\right|=$ $0)$, then $O(\sqrt{b / n}) \sqrt{n}\left|\hat{m}_{n}\left(\psi_{1}\right)\right|\left|\hat{m}_{n}\left(\psi_{2}\right)\right| \longrightarrow 0$ in probability as $n \longrightarrow \infty$. The last convergence follows from convergence of $\sqrt{n} \hat{m}_{n}\left(\psi_{1}\right)$ in distribution to Gaussian random variable (see Theorem 2.1 in Lenart, 2013), convergence $O(\sqrt{b / n})\left|\hat{m}_{n}\left(\psi_{2}\right)\right| \stackrel{p}{\longrightarrow} 0$ as $n \longrightarrow \infty$, the continuous mapping theorem and Slutsky's lemma. If $\left|m\left(\psi_{1}\right) m\left(\psi_{2}\right)\right| \neq 0$, then from Theorem 2.1 in Lenart and Pipień, 2013, the multivariate delta method and Slutsky's lemma we have that $O(\sqrt{b / n}) \sqrt{n}\left|\hat{m}_{n}\left(\psi_{1}\right) \hat{m}_{n}\left(\psi_{2}\right)-m\left(\psi_{1}\right) m\left(\psi_{2}\right)\right| \stackrel{p}{\longrightarrow}$ 0 as $n \longrightarrow \infty$. 


\section{Acknowledgments}

The authors would like to express their sincere gratitude to the anonymous referees for their valuable suggestions. Łukasz Lenart was supported by the Polish National Science Center based on decision number DEC2013/09/B/HS4/01945. Anna Dudek has received funding from the European Union's Horizon 2020 research and innovation programme under the Marie Skłodowska-Curie Grant Agreement No. 655394. 


\section{References}

[Bell et al., 2012] Bell, A., Holan, S., and McElroy, T. (2012). Economic Time Series: Modeling and Seasonality. Chapman and Hall/CRC.

[Corduneanu, 1989] Corduneanu, C. (1989). Almost Periodic Functions. Chelsea, New York.

[Hurd and Miamee, 2007] Hurd, H. and Miamee, A. (2007). Periodically Correlated Random Sequences: Spectral Theory and Practice. Wiley, Hoboken, New Jersey.

[Kim, 1994] Kim, T. (1994). Moment bounds for non-stationary dependent sequances. J. Appl. Prob., 31:731-742.

[Lenart, 2013] Lenart, Ł. (2013). Non-parametric frequency identification and estimation in mean function for almost periodically correlated time series. Journal of Multivariate Analysis, 115:252-269.

[Lenart et al., 2008] Lenart, Ł., Leśkow, J., and Synowiecki, R. (2008). Subsampling in testing autocovariance for periodically correlated time series. J. Time Ser. Anal., 29(6):995-1018.

[Lenart and Pipień, 2013] Lenart, Ł. and Pipień, M. (2013). Seasonality Revisited - statistical Testing for Almost Periodically Correlated Stochastic Processes. Central European Journal of Economic Modelling and Econometrics, 5(2):85-102.

[Napolitano, 2012] Napolitano, A. (2012). Generalizations of Cyclostationary Signal Processing: Spectral Analysis and Applicationss. Wiley-IEEE Press.

[Napolitano, 2016] Napolitano, A. (2016). Cyclostationarity: New trends and applications. Signal Process., 120:385-408.

[Politis et al., 1999] Politis, D., Romano, J., and Wolf, M. (1999). Subsampling. Springer-Verlag, New York. 